\title{
MINILAPAROTOMIAS PARA TRATAMENTO DE DOENÇAS DO CÓLON
}

\section{APROACH TO COLON DISEASES BY MINILAPAROTOMY}

\author{
Peretz Capelhuchnik, TCBC-SP'; Paulo de Azeredo Passos Candelária, TCBC-SP²; \\ Fang Chia Bin ${ }^{3}$; Wilmar Artur Klug ${ }^{4}$; Jorge Alberto Ortiz, TCBC-SP ${ }^{5}$
}

\begin{abstract}
RESUMO: Objetivo: Estudar as possibilidades técnicas e resultados obtidos com pequenas incisões para tratar doenças do cólon. Método: Vinte e seis pacientes, 12 do sexo masculino e 14 do feminino foram submetidos a laparotomias com incisões menores que $10 \mathrm{~cm}$. As minilaparotomias variaram entre 3,6 e $10 \mathrm{~cm}$ (média $-7,11 \mathrm{~cm}$ ), paramedianas e realizadas sobre a área tumoral localizada por palpação ou colonoscopia. Várias operações foram realizadas: hemicolectomias direitas, sigmoidectomias, colectomias segmentares e colotomias. As doenças tratadas foram: adenocarcinoma (20), lipoma (1), pólipo adenomatoso (1), carcinóide (1), Crohn íleo-cecal (1), adenoma viloso (1) e mucocele (1). Resultados: Vinte e cinco enfermos evoluíram sem complicações. Uma paciente de 90 anos, com câncer de cólon ocluído e disseminado faleceu com broncopneumonia e com função intestinal normal. As funções intestinais retornaram cedo: emissão de gases no $2^{\circ}$ e $3^{\circ}$ dias, evacuações no $3^{\circ}$ e $4^{\circ}$ dias. Conclusões: Tumores menores que $8 \mathrm{~cm}$ puderam ser facilmente operados através de minilaparotomias. Tumores maiores ou fixos não devem ser operados por pequenas incisões. Princípios oncológicos foram respeitados como na cirurgia convencional. A recuperação dos doentes foi confortável e sem queixa de dor significativa. O efeito cosmético foi excelente (Rev. Col. Bras. Cir. 2005; 32(4): 192-194).
\end{abstract}

Descritores: Neoplasias do cólon; Doenças do cólon; Laparotomia/métodos; Colectomia/métodos.

\section{INTRODUÇÃO}

As incisões mini-invasivas representam um refinamento técnico, e verdadeira arte em cirurgia colo-retal. Sua utilização tem vários objetivos: estética, menor trauma, menos dor, maior conforto pós-operatório e retorno precoce às atividades normais, o que é compartilhado por diversos autores $^{1-5}$.

Nosso entusiasmo com incisões mini-invasivas vem da década de 70, era pré-laparoscópica com o sucesso que obtivemos com minilaparotomias para colecistectomias. Com o advento da cirurgia laparoscópica e depois, a assistida, com incisões pequenas, achamos que com as mesmas e sem laparoscopia conseguiríamos resultados semelhantes ${ }^{6.7}$. Aos poucos percebemos que os casos deveriam ser selecionados e de preferência com a doença localizada em áreas privilegiadas: cólon direito, transverso e sigmóide que são mais facilmente mobilizáveis.

Em 1995 comunicamos nossos resultados preliminares no Congresso Brasileiro da Colo-proctologia ${ }^{1,2}$. Trata-se de atraente caminho alternativo à cirurgia convencional ou laparoscópica ${ }^{8,9}$. O conceito de minilaparotomia é discutido por vários autores $4,5,10,11$. Aceitamos como definição, assim como Furstemberg et al. ${ }^{4}$, incisões de até $10 \mathrm{~cm}$.

Nosso objetivo foi avaliar as possibilidades técnicas e resultados obtidos com minilaparotomias no tratamento de afecções cirúrgicas dos cólons.

\section{MÉTODO}

Foram estudados 26 doentes originários do Departamento de Cirurgia da Faculdade de Ciências Médicas da Santa Casa de São Paulo, em sua maioria, e alguns de clínica privada.

O preparo intestinal foi realizado com dieta sem resíduos e uso oral de Bisacodil e 1000ml de solução de lactose a 7,5\% iniciados antes da internação hospitalar.

O uso profilático de anti-bacteriamos foi realizado com cefalotina e metronidazol durante 24 horas e iniciados uma hora antes do ato cirúrgico.

O material cirúrgico utilizado foi o convencional. Usamos afastadores do tipo Faraboeuf ou Richardson que ocupam menos espaço. A Incisão da pele foi longitudinal, paramediana à direita ou esquerda (cólon direito ou esquerdo), sobre a área tumoral, localizada pela palpação ou colonoscopia. A pele era descolada para permitir maior mobilidade.

Incisões peritoneais do recesso parieto cólico direito permitem a mobilização completa do segmento ileo-cecal até angulo hepático e transverso. Esta mobilização é alternante, proximal e distal ao tumor. O segmento dissecado é reintroduzido na cavidade abdominal para mobilização do outro.

Da mesma forma, no sigmóide, as incisões nos folhetos do mesocólon de ambos os lados permite a sua mobilização. A exteriorização do intestino contendo o tumor e meso correspondente é suficiente e extensa para permitir uma

1. Prof. Titular do Departamento de Cirurgia da Faculdade de Ciências Médicas da Santa Casa de São Paulo.

2 . Coordenador da Área de Colonoscopia da Disciplina de Colo-proctologia do Departamento de Cirurgia da Irmandade da Santa Casa de Misericórdia de São Paulo; Mestre em Cirurgia.

3. Prof. Adjunto Doutor da Faculdade de Ciências Médicas da Santa Casa de São Paulo.

4. Prof. Titular do Departamento de Cirurgia da Faculdade de Ciências Médicas da Santa Casa de São Paulo.

5. Coordenador da Área de Fisiologia Anal da Disciplina de Colo-proctologia do Departamento de Cirurgia da Irmandade da Santa Casa de Misericórdia de São Paulo; Mestre em Cirurgia.

Recebido em: 10/01/2005

Aceito para publicação em: 01/06/2005

Conflito de interesse: nenhum

Fonte de financiamento: nenhuma

Trabalho realizado no Departamento de Cirurgia da Faculdade de Ciências Médicas da Santa Casa de São Paulo. 
ressecção satisfatória do ponto de vista oncológico, realizada fora da cavidade abdominal e permitindo a ligadura dos vasos em sua origem (Figura1).

As anastomoses íleo-cólicas ou colo-cólicas foram término-terminais e manuais. A drenagem foi realizada em apenas um caso que estava sub-ocluído e com disseminação tumoral. Nenhum paciente recebeu transfusão de sangue durante a operação ou no pós-operatório.

\section{RESULTADOS}

A faixa etária variou entre os extremos de 24 a 90 anos. A média de idade foi 67,46 anos. Dos 26 enfermos, 12 eram do sexo masculino e 14 do feminino.

As causas que indicaram a cirurgia foram variadas (Tabela 1) e as operações realizadas diversas (Tabela 2).

Apenas dois pacientes necessitaram de transfusões para correção de anemia, um diabético e um idoso.

Os resultados anatomo-patológicos dos espécimes estão representados na Tabela 1 e correspondem às indicações cirúrgicas. Vinte e cinco pacientes evoluíram sem complicações. Usamos analgésicos apenas nos dois primeiros dias.

A emissão de gazes ocorreu no $2^{\circ}$ ou $3^{\circ}$ dias. Evacuações aconteceram no $3^{\circ}$ e $4^{\circ}$ dias.

Uma paciente com 90 anos, sub-ocluída e com disseminação tumoral foi operada como ressecção paliativa. Teve broncopneumonia e faleceu apesar de ter sua função intestinal normalizada.

\section{DISCUSSÃO}

O conceito de minilaparotomia depende de opinião pessoal. Assim Fleshman et al. ${ }^{3}$ referem incisões entre oito e

Tabela 1 - Indicações cirúrgicas.

\begin{tabular}{ll}
\hline Diagnósticos & N.de pacientes operados \\
\hline Adenocarcinoma & 20 \\
& (Dukes): A-0; B-10; C-10. \\
Lipoma & 01 \\
Polipo Adenomatoso & 01 \\
Carcinóide & 01 \\
Adenoma Viloso & 01 \\
Crohn Ileocecal & 01 \\
Mucocele & 01 \\
Total & $\mathbf{2 6}$ \\
\hline
\end{tabular}

Tabela 2-Operações realizadas.

\begin{tabular}{lc}
\hline Operações realizadas & N. de pacientes operados \\
\hline Hemicolectomia Direita & 15 \\
Sigmoidectomia & 06 \\
Colectomia Segmentar & 02 \\
Colotomia & 02 \\
Exerese de Mucocele & 01 \\
Total & $\mathbf{2 6}$ \\
\hline
\end{tabular}

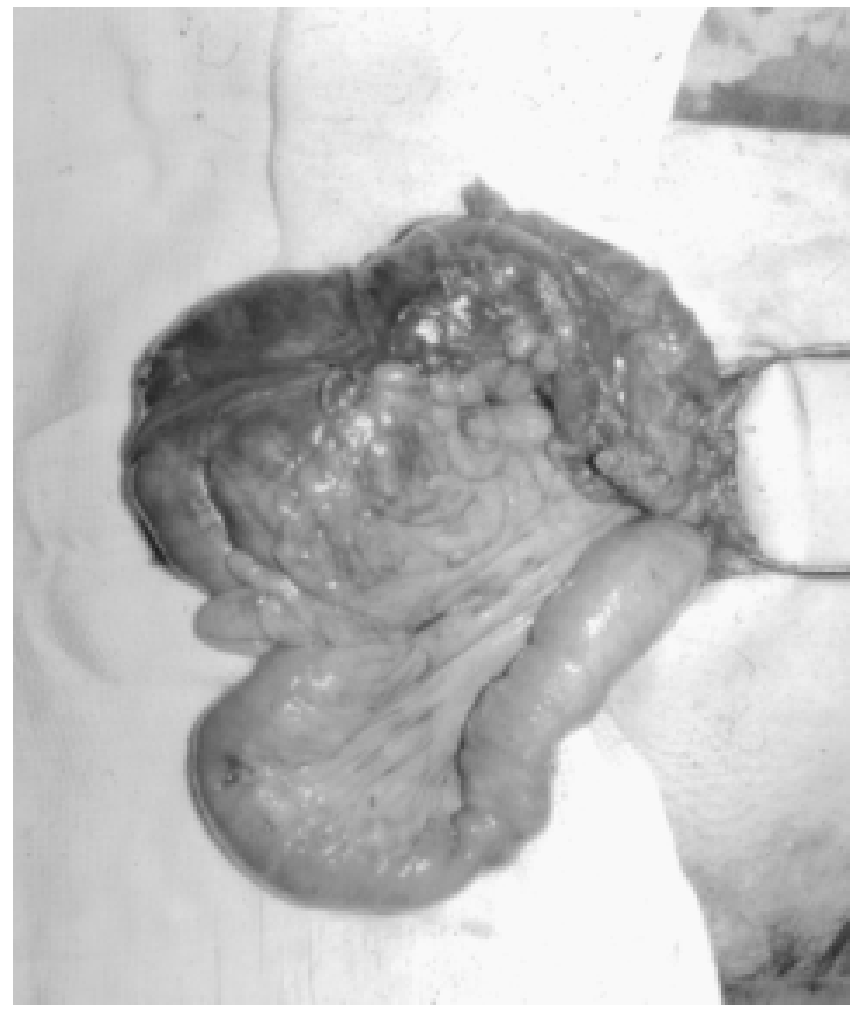

Figura 1 - Íleo terminal, cecum, ascendente e transverso exteriorizados através de incisão para-retal direita e infra-umbilical de 5,5 cm (Tumor de colon ascendente).

$18 \mathrm{~cm}$. Nakagoe et al. ${ }^{11}$ assim como Takegami et al. ${ }^{10}$ limitam até $7 \mathrm{~cm}$. Lechaux et al..$^{5}$ em suas minilaparotomias hipogástricas aceitam incisões até $12 \mathrm{~cm}$. Aceitamos o critério de Furstemberg et al. ${ }^{4}$ que consideram o limite até $10 \mathrm{~cm}$

As incisões por nós praticadas variaram entre 3,6 e $10 \mathrm{~cm}$ (média de $7,11 \mathrm{~cm}$ ). À medida que a experiência aumentou, conseguimos trabalhar com incisões menores. Estas incisões são competitivas com as convencionais ou laparoscópicas, mas tem limitações e devem ser seletivas. Os segmentos dos cólons que melhor permitem a sua ressecção com pequenas incisões são: cólon direito, transverso e o sigmóide. Estas áreas são privilegiadas pela sua mobilidade natural ou facilidade de mobilização. A exposição das vísceras através das minilaparotomias foi sempre ampla e nos casos de tumor maligno permitiu uma ressecção oncológica correta, englobando comprimento do cólon ressecado, bem como número de gânglios semelhantes aos obtidos na cirurgia convencional.

Conseguimos operar tumores de até $8 \mathrm{~cm}$ de comprimento. Em duas ocasiões tivemos a frustração de não conseguir a exteriorização dos tumores sem ampliar a incisão apesar de amplamente mobilizados. O mesmo ocorreu com tumores fixos. Estes pacientes foram excluídos do trabalho. Achamos que tumores grandes, maiores que $8 \mathrm{~cm}$, fixos ou disseminados não podem ser operados através de minilaparotomias. Não tivemos experiência com pacientes obesos.

Um dos pacientes, oito meses após a cirurgia apresentou-se com tumor em outro segmento do cólon. Acreditamos tratar-se de tumor sincrônico, e que, estando sub-ocluído o anteriormente operado, não permitiu a passagem do colonoscópio 
para detectá-lo. Colonoscopias ou enemas baritados devem ser obrigatoriamente realizados no pré-operatório, bem como estadiamento com tomografia computadorizada ou ressonância magnética para detecção de tumores síncrônicos ou metástases.

Nakagoe et al.$^{12}$ referem preocupação com a possibilidade de infecção ou implante de células cancerosas na incisão. Estas ocorrências não aconteceram em nenhum dos nossos casos. Admitimos que procuramos evitar o contacto do tumor com a incisão bem como no final da cirurgia lavá-la com soro fisiológico e antisséptico.

A incidência de eviscerações nas laparotomias convencionais varia entre 0,3 e $2,58 \%$ tem significativa mortalidade 5 . Em 76 laparotomias convencionais para cura de megacólon tivemos três $(3,95 \%)$ eviscerações sem mor- talidade $^{13}$. Em minilaparotomias referidas por diversos autores $^{1,3-5}$ e em nossa casuística esta complicação não ocorreu.

O custo das operações por minilaparotomias exclusiva não pode ser comparado ao das laparoscópicas, bastante elevado, e que exige tempo maior, equipamentos especiais e materiais descartáveis ${ }^{8,14}$.

Este estudo permitiu as seguintes conclusões:

1) A recuperação foi confortável, sem queixa de dor significativa

2) Houve retorno precoce da função intestinal

3) A minilaparotomia permitiu ressecção oncológica semelhante à obtida na cirurgia convencional

4) $\mathrm{O}$ efeito cosmético foi excelente.

\begin{abstract}
Background: To study the technical possibilities and results of the minilaparotomy approach to colon diseases. Methods: Twenty-six patients, 12 males and 14 females were submitted to laparotomies with skin incisions smaller than 10cm length. Incisions were paramedian over the tumoral area, localized by palpation or colonoscopy. Results: Minilaparotomies extension varied between 3.6 and $10 \mathrm{~cm}$ (average $-7.11 \mathrm{~cm}$ ) to treat colon diseases. Several operations were performed through minilaparotomies as follows: right colectomies, sigmoidectomies, segmental colectomies and colotomies. The diseases treated by minilaparotomies were: adenocarcinoma - 20, lipoma - 1, adenomatous polyp - 1, carcinoid - 1, ileocecal Crohn - 1, villous adenoma - 1 and mucocele - 1. Twenty-five patients didn't have complications. A 90 years old woman with an occluded and disseminated sigmoid cancer died from bronchopneumonia in spite of normal intestinal function. Bowel function was restored very early with passage of flatus during the second and third days and stools during the third and fourth days. Tumors $8 \mathrm{~cm}$ length or smaller could be handled by small incisions, larger or fixed tumors could not be treated by minilaparotomies. Conclusion: Minilaparotomies approach to treat colon diseases seems to be an alternative procedure to conventional or laparoscopic surgery in selected cases. The oncologycal principles and resection for colon cancer are the same as obtained with conventional laparotomies. Recovery was uneventful and without significant pain. It represents a suitable technique with a superior cosmetic effect.
\end{abstract}

Key words: Colonic Neoplasms; Colonic diseases; Laparatomy/methods; Colectomy/methods.

\section{REFERÊNCIAS}

1. Capelhuchnik P, Klug WA, Fang CB, et al. Minilaparotomias para cirurgia do cólon: estudo preliminar. Rev Bras Coloproctol.1995;15(supl. 1)

2. Capelhuchnik P. Minilaparotomias: comentário editorial. Rev Bras Coloproctol. 1999;19(1):63

3. Fleshman JW, Fry RD, Birnbaum EH, et al. Laparoscopic assisted and minilaparotomy approaches to colorectal diseases are similar in early outcome. Dis Colon Rectum.1996;39(1):15-22.

4. Fürstenberg S, Godman S, Machado M. Minilaparotomy approach to tumors of the right colon. Dis Colon Rectum. 1998;41(8):997-9.

5. Lechaux JP, Gerbaux B, Lambert MP, et al. Incision de Pfannenstiel en chirurgie colo-rectale. Chirurgie. 1997;122(7):418-23.

6. Jager RM, Wexner DS, editors. Laparoscopic colorectal surgery. $1^{\text {st }}$ ed. New York: Churchill Livingstone; 1996.

7. Khalili TM, Fleshner PR, Hiatt JR, et al. Colorectal cancer: comparison of laparoscopic with open approches. Dis Colon Rectum. 1998;41(7):832-8.

8. Marcello PW, Milsom JW, Wong SK, et al. Laparoscopic restorative proctocolectomy: case matched comparative study with open restorative proctocolectomy. Dis Colon Rectum. 2000;43(5):604-8.
9. Ou H. Laparoscopic assisted mini laparotomy with colectomy. Dis Colon Rectum. 1995;38(3):324-6

10. Takegami K, Kamaguchi Y, Nakayama H. Minilaparotomy aproach to colon cancer. Surg Today. 2003;33(6):414-20.

11. Nakagoe T, Sawai T, Tsuji T, et al. Use of minilaparotomy in the treatment of colonic cancer. Br J Surg. 2001; 88(6):831-6.

12. Nakagoe T, Sawai T, Tsuji T, et al. Minilaparotomy wound edge protector (Lap-Protector): a new device. Surg Today. 2001;31(9):850-2.

13. Capelhuchnik P. Tratamento do megacólon pela hemicolectomia esquerda [dissertação]. São Paulo (SP): Faculdade de Ciências Médicas da Santa Casa de São Paulo; 1975.

14. Brown SR, Eu KW, Seow-Choen F. Consecutive series of laparoscopic - assisted vs. minilaparotomy restorative proctocolectomies. Dis Colon Rectum. 2001; 44(3):397-400

Endereço para correspondência:

Peretz Capelhuchnik

Rua José Maria Lisboa n. 1370 apt. $7^{\circ}$ andar

Cerqueira César

01423-001 - São Paulo - SP

E-mail: peretz@terra.com.br 